\title{
Estrogen Receptor preparation effects on the receptor-DNA interaction by surface plasmon resonance.
}

Sandrine Bayle ${ }^{1}$, Joel Chopineau ${ }^{2,4}$, Benoit Roig ${ }^{2,3}$, Denis Habauzit 1, 5, 6

1) Laboratoire Génie de l'Environnement Industriel, Ecole des Mines d'Alès, 6 Avenue de Clavières, 30319 , Alès Cedex, France.

2) Université de Nîmes, F-30021 NIMES, France

3) Université de Nîmes, EA7352 CHROME, Rue Georges Salan, 30021 Nîmes, France

4) Institut Charles Gerhardt Montpellier, UMR 5253 CNRS-ENSCM-UM Ecole Nationale Supérieure de Chimie, Montpellier, France

5) Université de Rennes 1, F-35043 RENNES, France

6) Institut national de la santé et de la recherche médicale (Inserm), Institut de recherche en santé, environnement et travail (Irset - Inserm UMR 1085), F-35000 RENNES, France

\section{Correspondence}

Dr D. Habauzit

IRSET, Institut de Recherche en Santé, Environnement et Travail, Université de Rennes 1, EHESP, Equipe "TREC"- 9 avenue du Professeur Léon Bernard, 35000 RENNES cedex, France

Phone: +33-2 23239053

E-mail : denis.habauzit@univ-rennes1.fr

Surface plasmon resonance, Estrogen, Estrogen receptor, Dimerization, Temperature 


\begin{abstract}
Up to now several studies have investigated estrogen receptor (ER) / estrogen response element (ERE) interaction using biosensors such as surface plasmon resonance. These strategies have aimed to understand the molecular mechanism of such interaction as well as the effect of the ligand on this interaction. These approaches start to be used to determine the mechanisms of protein / DNA interaction, in particular in the context of drug discovery or environmental applications. However some physical and biochemical parameters (incubation time, temperature, protease inhibitor cocktail and BSA...) are not completely described in the literature and could deeply modify the obtained results. This paper aims to focus on the preliminary steps of sample preparation such as protein thawing, incubation conditions (time and temperature) but also on the evaluation of protease inhibitor cocktail and BSA effect in the measurement of ER/ERE interactions.
\end{abstract}




\section{Introduction}

Since three decades several publications have aimed to understand the molecular mechanisms by which estrogen receptor alpha $(\mathrm{ER} \alpha)$ bind with small palindromic consensus sequence known as estrogen response element (ERE) in the presence of the ER natural ligand 17- $\beta$-estradiol (E2) [1-3]. The first assessments were mainly based on the use of band shift, and then the emergence of new technologies, such as surface plasmon resonance [4], fluorescence anisotropy [5, 6], quartz crystal microbalance [7], gold nanoparticles [8], silicon nanowire [9], have permit to characterize the interaction properties of ER $\alpha$ towards ERE. Moreover knowledge in E2/ER/ERE interaction is used for the development of new screening methods in drug discovery [10] and also for the monitoring the presence of endocrine disrupting chemicals (EDC) in environment $[11,12]$.

Previous papers have described ER/ERE interaction for several purposes [13] by using Surface Plasmon Resonance as analytical tool. The first one is the molecular mechanism by which ER bind with E2 [10, 12], form a dimer $[11,14]$ and then interact alone or with cofactor on ERE [4, 11, 15-17]. This knowledge was used for the determination of interaction properties (i.e interaction constant) and recently for environmental purpose (i.e detection or quantification) $[11,18,19]$. But a discrepancy in the experimental conditions reported in the literature can be noticed [20] and few publications have detailed the biochemical parameters used and the influence of the parameters choice.

On the whole, this study aims to show the outstanding of ER preparation initial steps, generally not described in the literature, but that were found very fundamental for a reproducibility of the analytical method. Three steps are necessary, ER is thawed, then ER is mixed with E2, and finally ER/E2 is injected for interaction with ERE. An overnight incubation at $4{ }^{\circ} \mathrm{C}$ is required for ER dimerization [11]. In our previous paper, we compared various experimental conditions usually employed for the determination of ER/ERE interaction properties [20] such as the effects of solvent in the estrogenic compounds solubilisation, the temperature and the time for the E2/ER incubation. Three parameters could deeply modify the ER binding level towards ERE [20]. A decrease of ER/ERE binding level was clearly observed depending on the temperature and time. Furthermore, incubation at different times and temperatures induce a decrease of the ER binding level, due to a thermal deactivation of the active ER form. In this context, the present paper aims to study the influence of the temperature of ER thaw and the presence of two additional compounds, which are known to avoid protein damages (bovine serum albumin (BSA) and protease inhibitor cocktail). These two last assessed parameters have 
(1) potential aspecific interaction with E2 and the surface or prevention of ER enzymatic degradation by competition (BSA) and have (2) prevent ER enzymatic degradation by competition and involvement in ER stability (protease inhibitor cocktail).

\section{Materials and methods}

\section{Reagents}

Chemicals, Tris buffer, tween 20, methanol $17 \beta$-estradiol (E2), $\mathrm{NaCl}, \mathrm{MgCl}_{2}$, bovine serum albumin (BSA) and EDTA free protease inhibitor cocktail for use with mammalian cell and tissue extract were analytical grade and purchase from Sigma-Aldrich (Saint Quentin Fallavier, France). Human recombinant estrogen receptor alpha (ER $\alpha)$, and estrogen response element (ERE) sequence were obtained as previously described [11].

\section{ER preparation and incubation period in ice or ambient temperature}

$\mathrm{ER} \alpha$ was stored at $-80^{\circ} \mathrm{C}$ until their use and was then thaw on ice or at ambient temperature $\left(22{ }^{\circ} \mathrm{C}\right)$ during 1 hour (Fig. 1). ER stock solution was diluted in TNMT buffer $(50 \mathrm{mM}$ Tris $\mathrm{HCl}, 150 \mathrm{mM} \mathrm{NaCl}, 10 \mathrm{mM}$ $\mathrm{MgCl}_{2}, 0.05 \%$ Tween $20, \mathrm{pH} 7.5$ ) to a final concentration of $100 \mathrm{nM}$, and then equally mix were stored either on ice or at ambient temperature for one hour (Fig. 1) with TNMT containing different concentrations of E2 previously dissolved in methanol. Methanol concentration was $0.1 \%$ in TNMT buffer. The final concentration of ER and $\mathrm{E} 2$ was $50 \mathrm{nM}$ and between $1 \mathrm{nM}$ and $100 \mathrm{nM}$ respectively.

\section{Preparation of BSA and protease inhibitor solution and mixing step with ER}

BSA and anti-protease cocktail were prepared in TNMT buffer. ER solution (with or without E2) was prepared with anti-protease cocktail (1/2000 of TNMT) or BSA (final concentration of $1 \mathrm{mg} / \mathrm{ml}$ ). Finally all ER preparations were incubated at least overnight before their analysis on Biacore instrument. 


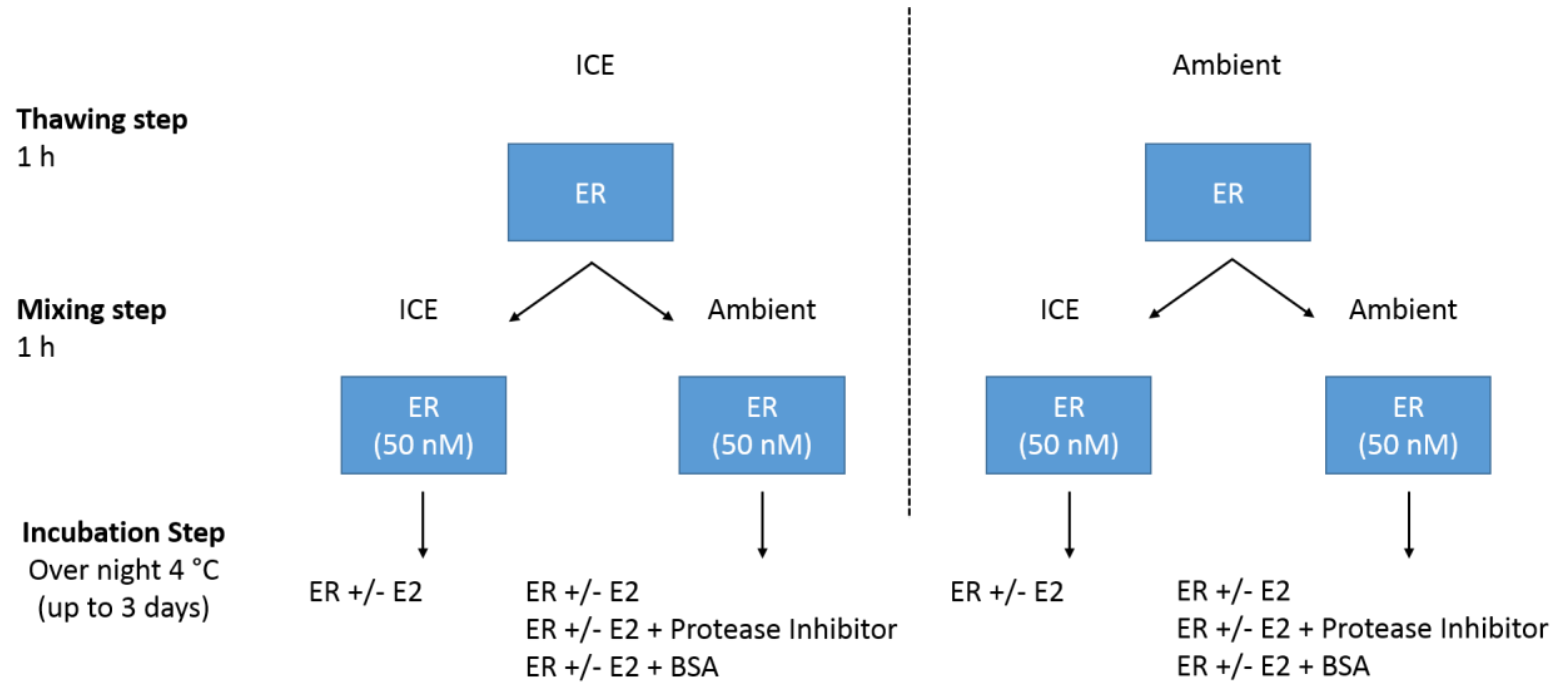

Fig. 1. Summary of experiment realized

\section{ERE preparation and binding on Sensor chip SA}

Double stranded biotinylated estrogen response element (ERE) was diluted in TNMT buffer up to a concentration of $450 \mathrm{nM}$ and then injected with a flow rate of $5 \mu 1 / \mathrm{min}$ in Biacore apparatus. Then $770 \mathrm{RU}$ of ERE was immobilized on Sensor Chip SA (Biacore) previously activated by two injections of $\mathrm{NaOH}$ solution $(50 \mathrm{mM})$ and then one injection of SDS $(0.1 \%)$.

\section{SPR experiments}

Experiments were performed at $25{ }^{\circ} \mathrm{C}$ on Biacore 1000 apparatus. After ERE immobilisation, ER samples were injected on the sensor chip with a flow rate of $20 \mu 1 / \min$ during $2 \mathrm{~min}$. After the injection the dissociation phase was followed during $180 \mathrm{~s}$. ER binding level was measured $24 \mathrm{~s}$ after each injection. The surface was regenerated with $0.1 \%$ of SDS solution followed by 2 min in running buffer. 


\section{Results}

\section{Incubation temperature effects on the ER/ERE binding level}

During manipulations implementing the ER / E2 / ERE interaction, several steps were required to set the temperature and time such as the ER thawing step and the incubation step of ER $\alpha$ protein with E2. The effect of these steps was evaluated by the measurement of the ER binding level on ERE in the presence of E2 concentration ranging from $10^{-9}$ to $10^{-7} \mathrm{M}$. As shown in Fig. 2, modification of temperature during these steps modify the ER binding in the presence of low concentration of E2 $\left(10^{-9} \mathrm{M}\right)$ with an average of $1247 \pm 13 \mathrm{RU}$ (mixing ambient) and $989 \pm 9$ RU (mixing ice) for ER protein thaw on ice and $669 \pm 26 \mathrm{RU}$ (mixing ambient) and $789 \pm 53 \mathrm{RU}$ (mixing on ice) for protein thaw at ambient temperature respectively (Fig. 2). Such discrepancies are also observed for high E2 concentration $\left(10^{-7} \mathrm{M}\right)$ with an average of $2097 \pm 109$ RU (mixing ambient) and $1232 \pm 91 \mathrm{RU}$ (mixing ice) for ER protein thaw at ambient temperature and $1131 \pm 80 \mathrm{RU}$ (mixing ambient) and $1154 \pm 97 \mathrm{RU}$ (mixing on ice) for protein thaw on ice respectively. Three conditions (thawing ice/mixing ice; thawing ambient/mixing ice; thawing ambient/mixing ambient) reach the same level of ER binding in the presence of high concentration of E2. Considering the ratio of the signal ER with $10^{-7} \mathrm{M}$ E2 / signal ER with $10^{-9} \mathrm{M}$ E2 increase due to the increase of E2 concentration, the values are respectively 1.7, 1.2, 1.7 and 1.5 (Fig. 2 A-B) depending upon the step used.

Thawing ER protein on ice instead of ambient temperature induces a higher level of the basal ER binding level (Fig. 2A). But these differences are less pronounced for higher E2 concentration; the influence of the presence of E2 in the sample is lower when incubation was performed on ice, the ratio reaches 1.2 and 1.5 instead of 1.7 for room temperature incubation. Previously, high concentration of E2 was showed to be linked with ER dimerization [11]. The whole results ask on the ER state during the experiment and the ER binding level may not represent the same event as previously published. As the ER binding level decreases with the temperature used during the sample preparation, the question of the level of degradation of the protein ER is raised at ambient temperature is worth mentioning. 
A
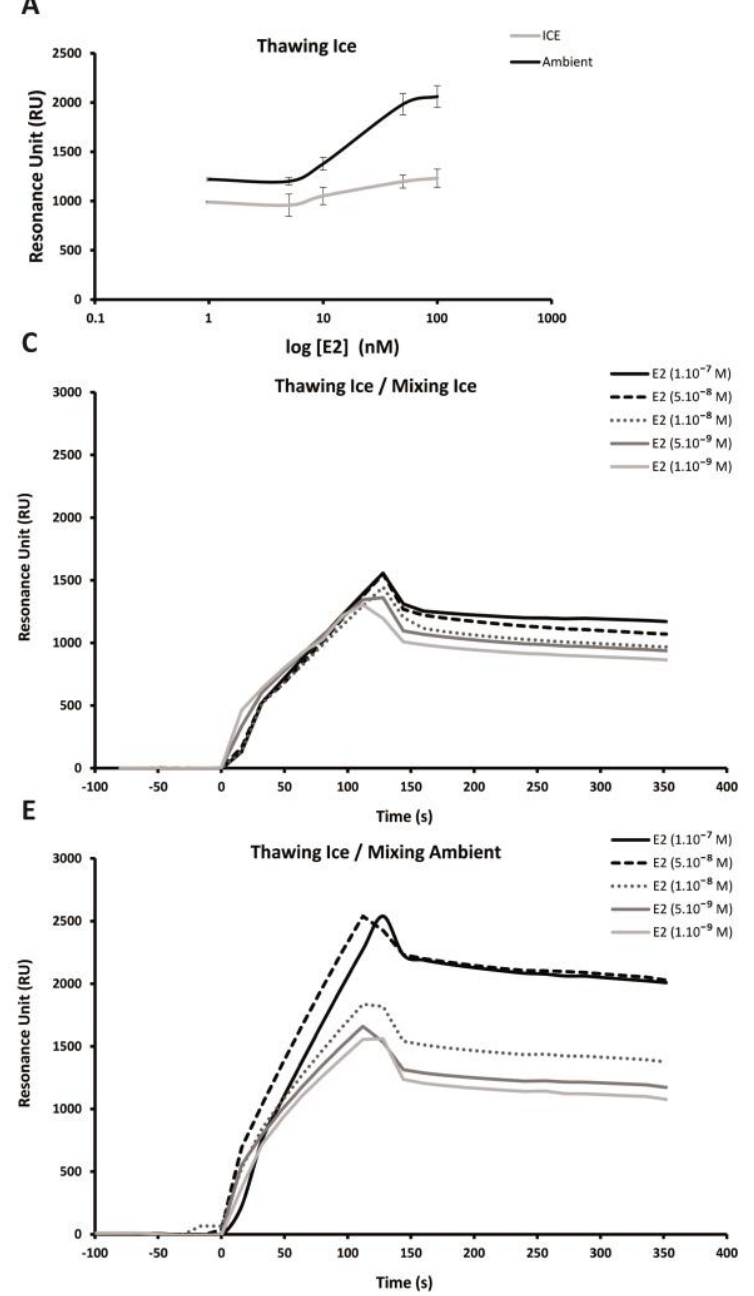

B
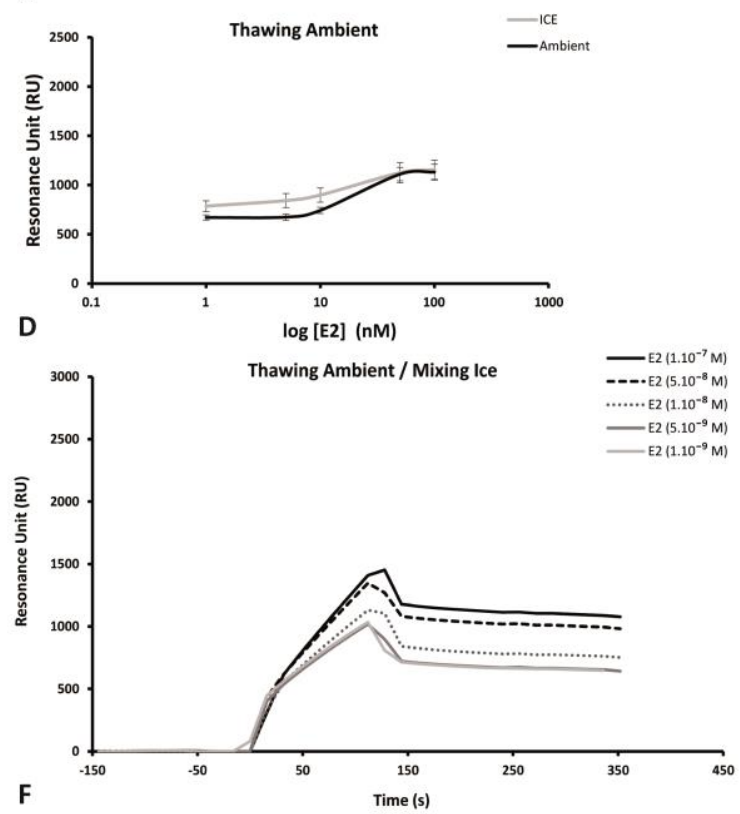

F

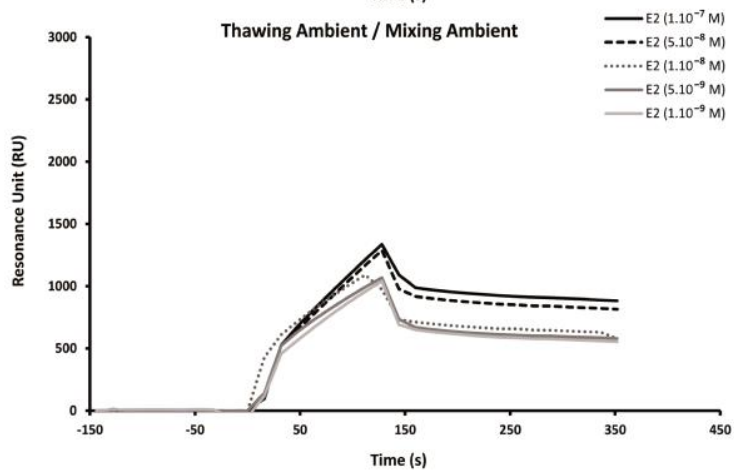

Fig. 2. Thawing and mixing temperature effect on ER binding level. Mean of the ER binding level after thawing onto ice (A) or thawing at ambient temperature (B). (C) to (E): Temporal sensor response to sample containing different concentrations of E2; thawing on ice - Mixing ice (C) or mixing at ambient temperature (E) and thawing at ambient temperature - mixing ice (D) or mixing ambient (F). The data are shifted along the X-axis.

\section{Effect of Protease inhibitor and BSA on ER/ERE binding level}

No clear difference in the ER binding profiles is observed between thaw ice / mix ice and thaw ambient / mix ice. It may be due to the fact that during the ambient thaw step the temperature is not ambient but between $-80^{\circ} \mathrm{C}$ and ambient temperature (Fig. 3). On the contrary, the combination with the second temperature step seems to be critical for ER binding level. Indeed, the ambient temperature after initial thaw step at ambient temperature produces a decrease of the ER binding level in the absence of E2 (Fig. 3 A-B). This decrease of around $25 \%$ is in the same order as those previously described after incubation at $21^{\circ} \mathrm{C}$ during two hours [20].

This decrease seems to demonstrate the thermal inactivation of ER is characterized by a decrease in the ER binding level. Moreover, the presence of a low amount of E2 prevents ER from this inactivation phenomena 
probably by maintaining ER in its active conformation. Indeed, high E2 concentration permits ER dimerization after overnight incubation (characterized by ligand dependent increase in ER binding level). Therefore the decrease of ER binding level on ERE after an ambient thawing could be linked either to a degradation of ER by proteolysis or by the influence of temperature on the protein folding. That decrease in binding level is never observed when ER is conserved on ice. Therefore, protease inhibitor cocktail and/or BSA were added to ER solutions (Fig. 3). The incubation was then performed in the same conditions.

The addition of protease inhibitor cocktail decreases the ER binding on ERE sequence. However, E2 induced an increase of the ER binding ratio that remain in the same range as after ice incubation with respectively 1.2 (Thawing ice /mixing ambient) and 1.5 (Thawing ambient /mixing ambient). Even if the protease inhibitor cocktail is EDTA free, the cocktail composition deeply prevents ER from binding to ERE as well as the ligand dependent dimerization of ER. The presence of BSA did not modify the ER binding level when the protein is incubated at ambient temperature regardless of the E2 concentration. The addition of BSA did not increase the SPR signal showing that there are neither interaction between ER and BSA nor aspecific BSA binding on the surface.
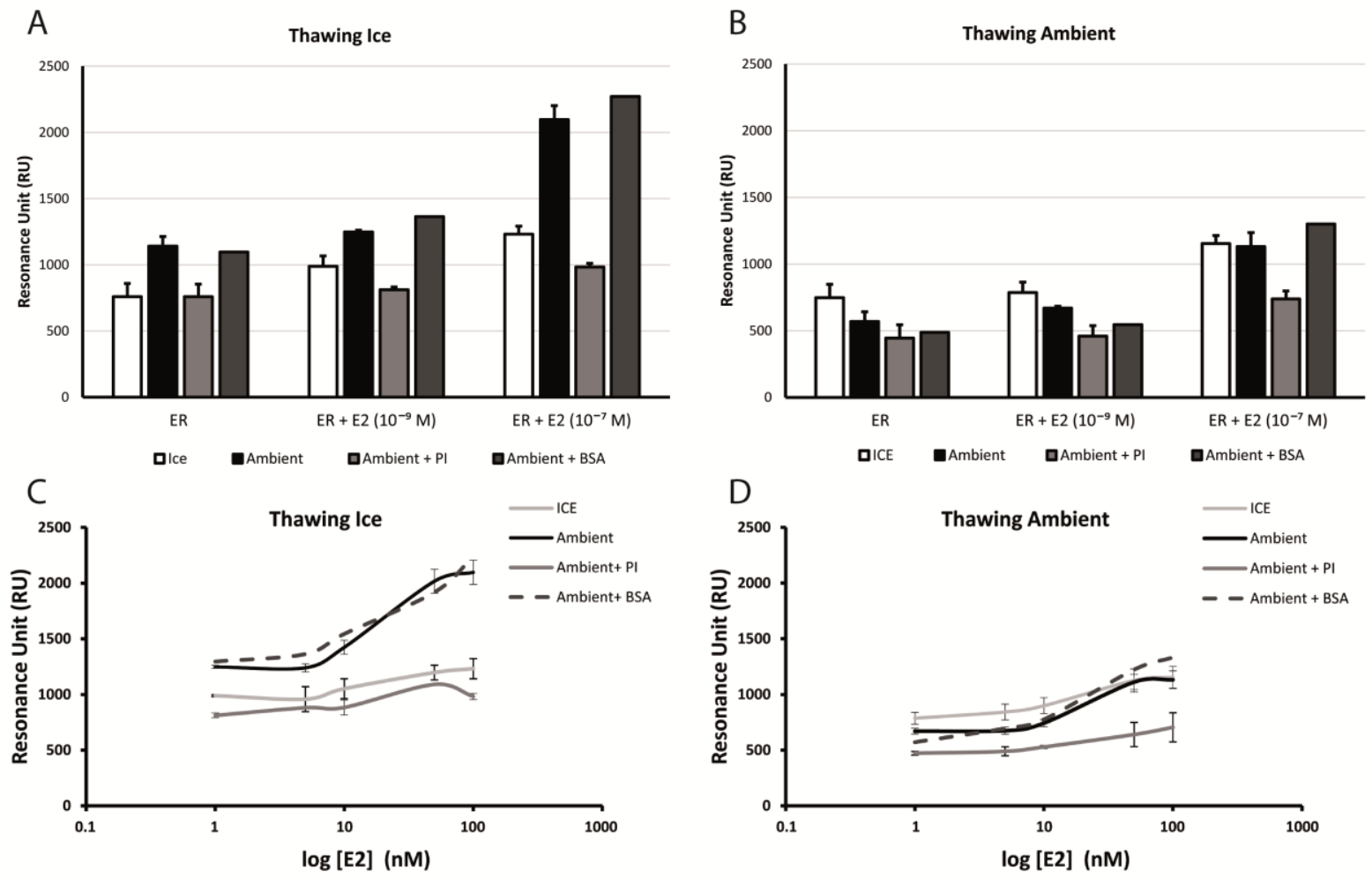
Fig. 3. Effect of conditions on the ER binding level onto ERE, either by comparison of the ER without E2 or with low or high concentration of E2 $(\mathrm{A}-\mathrm{B})$ or by studying the E2 dose effect on the ER binding level $(\mathrm{C}-\mathrm{D})$.

\section{Discussion}

Dimerization of ER is obtained in the presence of high concentrations of E2, but a decrease of the ER binding level was observed as a consequence of a thermal inactivation of ER [20]. Therefore, to decipher the role of time and thermal effect on ER activity, two critical parameters were assessed. The first one is the temperature of ER aliquot thaw. The second parameter is the temperature during ER/E2 mixing. The thawing step in ice and the mixing step at ambient temperature are necessary to obtain a high ER binding level. Then ER have to be incubated overnight at $4^{\circ} \mathrm{C}$. The other conditions, ER stabilization with protease inhibitor cocktail or BSA, were also studied to show if the decrease of ER binding level is not linked to the ER enzymatic degradation. Indeed these two substances could inhibit the enzymatic degradation of ER.

Except the protease inhibitor treated samples, all samples showed an increase of the ER binding level depending on E2 concentration $[11,20]$. This phenomena illustrates the importance of the $4^{\circ} \mathrm{C}$ overnight incubation that permits the dimerization process to occur. But the ER binding level magnitude as well as the ratio (ER high E2/ ER low E2) are linked with the temperature step occurring before the overnight incubation at $4^{\circ} \mathrm{C}$. Less difference is observed for the sample thawed on ice and mixed at ambient temperature. Here the ambient mix seems to improve the ER binding capacity by a $60 \%$ increase of the binding level. It may illustrate the ER refolding by the combination of the temperature and the overnight incubation. And low concentration of E2 seems to mimic this effect by increasing ER binding level by $25 \%$ for samples thawed on ice and incubated on ice. Conversely, for ambient incubation conditions no differences were observed with or without low concentration of E2. Interestingly, anti-protease cocktail inhibit drastically the ER binding level. It is merely due to one component of the anti-protease cocktail that modifies the ER dimerization and binding, unlike BSA that did not modify the binding level. These two conditions underline that the decrease of the ER binding level is related to the temperatures of thawing and of mixing and not to an enzymatic degradation.

\section{Conclusion}

ER binding level to ERE depends on the physical and biochemical parameters used for the preparation of the samples and those used during the interaction measurements. Up to now, no works have tried to compare 
these parameters effects on the protein binding level on ERE sequence. Here we have shown that these conditions (temperature used for ER thaw, temperature used for the mixing step and adjunction of BSA and protease inhibitor cocktail) modify interactions and underline that some other effects could affect the protein binding such as thermal inactivation as previously shown. Therefore, measurement of affinity constants should not been performed before the critical biochemical parameters established. Indeed, our data showed that depending upon the protocol used for the study of ER binding properties, the dimerization and thermal denaturation could be equally studied making difficult to determine what phenomena are measured. In conclusion, for biosensor development the best parameters are the ER thaw in ice followed by incubation at ambient temperature without BSA or protease inhibitor cocktail. Finally such biochemical parameters therefore deeply modify biosensor answer.

\section{Compliance with ethical standards}

Conflict of interest: The authors declare that they have no competing interest

\section{References}

1. Druege PM, Klein-Hitpass L, Green S, Stack G, Chambon P, Ryffel GU (1986) Introduction of estrogenresponsiveness into mammalian cell lines. Nucleic Acids Res 14:9329-9337. doi: 10.1093/nar/14.23.9329

2. Klinge CM (2001) Estrogen receptor interaction with estrogen response elements. Nucleic Acids Res 29:2905-2919.

3. Kumar V, Chambon P (1988) The estrogen receptor binds tightly to its responsive element as a ligandinduced homodimer. Cell 55:145-156. doi: 10.1016/0092-8674(88)90017-7

4. Cheskis BJ, Karathanasis S, Lyttle CR (1997) Estrogen Receptor Ligands Modulate Its Interaction with DNA. J Biol Chem 272:11384-11391.

5. Ozers MS, Hill JJ, Ervin K, Wood JR, Nardulli AM, Royer CA, Gorski J (1997) Equilibrium binding of estrogen receptor with DNA using fluorescence anisotropy. J Biol Chem 272:30405-30411.

6. Szatkowski Ozers M, Hill JJ, Ervin K, Royer CA, Gorski J (2001) The dissociation rate of estrogen receptor alpha from the consensus estrogen response element. Mol Cell Endocrinol 175:101-109.

7. Su X, Lin C-Y, O’Shea SJ, Teh HF, Peh WYX, Thomsen JS (2006) Combinational application of surface plasmon resonance spectroscopy and quartz crystal microbalance for studying nuclear hormone receptorresponse element interactions. Anal Chem 78:5552-5558. doi: 10.1021/ac0606103

8. Tan YN, Su X, Liu ET, Thomsen JS (2010) Gold-nanoparticle-based assay for instantaneous detection of nuclear hormone receptor-response elements interactions. Anal Chem 82:2759-2765. doi: 10.1021/ac9026498

9. Zhang G-J, Huang MJ, Luo ZHH, Tay GKI, Lim E-JA, Liu ET, Thomsen JS (2010) Highly sensitive and reversible silicon nanowire biosensor to study nuclear hormone receptor protein and response element DNA interactions. Biosens Bioelectron 26:365-370. doi: 10.1016/j.bios.2010.07.129 
10. Rich RL, Hoth LR, Geoghegan KF, Brown TA, LeMotte PK, Simons SP, Hensley P, Myszka DG (2002) Kinetic analysis of estrogen receptor/ligand interactions. Proc Natl Acad Sci U S A 99:8562-8567. doi: 10.1073/pnas.142288199

11. Habauzit D, Armengaud J, Roig B, Chopineau J (2008) Determination of estrogen presence in water by SPR using estrogen receptor dimerization. Anal Bioanal Chem 390:873-883. doi: 10.1007/s00216-007$1725-\mathrm{x}$

12. Seifert M, Haindl S, Hock B (1998) In vitro analysis of xenoestrogens by enzyme linked receptor assays (ELRA). Adv Exp Med Biol 444:113-117.

13. Habauzit D, Chopineau J, Roig B (2007) SPR-based biosensors: a tool for biodetection of hormonal compounds. Anal Bioanal Chem 387:1215-1223. doi: 10.1007/s00216-006-0958-4

14. Jisa E, Jungbauer A (2003) Kinetic analysis of estrogen receptor homo- and heterodimerization in vitro. J Steroid Biochem Mol Biol 84:141-148.

15. Li Z-L, Ueki K, Kumagai K, Araki R, Otsuki Y (2014) Regulation of bcl-2 transcription by estrogen receptor- $\alpha$ and c-Jun in human endometrium. Med Mol Morphol 47:43-53. doi: 10.1007/s00795-0130043-y

16. Song HY, Sun W, Prabhakar S, Aung KMM, Su X (2013) Study sequence rules of estrogen receptor $\alpha$ DNA interactions using dual polarization interferometry and computational modeling. Anal Biochem 433:121-128. doi: 10.1016/j.ab.2012.10.021

17. Song HY, Su X (2014) Spacer effect of cooperative binding of estrogen receptor $\alpha$ and specificity protein 1 to composite DNA: A surface plasmon resonance study. Sens Actuators B Chem 195:635-642. doi: 10.1016/j.snb.2014.01.069

18. Gao Y, Li X, Guo L-H (2013) Assessment of estrogenic activity of perfluoroalkyl acids based on ligandinduced conformation state of human estrogen receptor. Environ Sci Technol 47:634-641. doi: 10.1021/es304030x

19. Wong W-L, Chow C-F (2015) A new bisphenol A derivative for estrogen receptor binding studies with surface plasmon resonance. Environ Toxicol Chem SETAC 34:1390-1396. doi: 10.1002/etc.2939

20. Habauzit D, Bayle S, Benimelis D, Chopineau J, Roig B (2014) Impact of biochemical design on estrogen receptor/estrogen response element interaction by surface plasmon resonance technology. Arch Biochem Biophys 541:61-66. doi: 10.1016/j.abb.2013.11.006 